\title{
Modified ridge splitting technique using conical space main-tainers for dela- yed implant placement in highly atrophic maxillae.
}

\author{
Guillermo Cabanes-Gumbau ${ }^{1}$, Francisco Javier Silvestre ${ }^{2}$
}

${ }^{1}$ DDS, PhD. University Specialist in Implantology (U.I.B.). Member of the Spanish Society of Implants.

${ }^{2}$ MD, DDS, PhD. Assistant Professor of Stomatology, Department of Stomatology, University of Valencia. Head of the Stomatology Unit, Dr. Peset University Hospital (Valencia), Spain.

Correspondence:

Prof. F. J. Silvestre

Department of Stomatology

University of Valencia

Calle Gascó Oliag 1

46010-Valencia. Spain.

\begin{abstract}
Background: A low-morbidity surgical technique is described for the horizontal augmentation of highly atrophic alveolar ridges in which first surgical step implant placement is contraindicated. The aim of this case report was to present an alternative treatment for the rehabilitation of the atrophic maxilla.

Methods: The technique involves a crestal corticotomy with transverse expansion of the vestibular and lingual cortical layers, followed by the placement of threaded titanium space maintainers between the expanded bone tables.

Results: The resulting surgically created biological space within the residual socket is completely filled with blood of marrow origin and great osteogenic potential. Due to the preserving effect of the titanium maintainers, we avoid partial collapse of the ridge widening initially obtained, which tends to occur to one degree or other as a consequence of reabsorption during the physiological tissue repair process.

Conclusions: This type of bone regeneration requires no autologous bone harvesting from other intra- or extraoral donor zones, thereby avoiding the increased morbidity associated with such procedures. It appears that alveolar ridge augmentation through corticotomy and threaded space maintainers may be a viable treatment approach for the implants placement in the severely atrophied maxilla.
\end{abstract}

Key words: Alveolar ridge augmentation, osseous space maintainers. 


\section{Introduction}

Implant-based tooth replacement has become a widely requested treatment option among patients. As a result, the bone regeneration techniques often associated with such treatment are increasingly com-mon and are evolving with a view to ensuring maximum predictability with the least possible morbid-ity $(1,2)$.

In the concrete case of implant beds in very narrow alveolar ridges, different regenerative techniques have been developed, such as en bloc overlay autografts, the use of particulate biomaterial (autologous or heterologous) in combination with membranes (absorbable or otherwise), and the use of expansive corticotomies to separate the vestibular and lingual cortical layers through the production of green-stick fractures (3-5).

All these techniques can be combined and used to complement each other. In addition, they can be accompanied by immediate or delayed implant placement over the regenerated zone.

In general, in the case of severe horizontal atrophy, and regardless of the regenerative technique used, most authors recommend delayed implant placement in a second surgical step, since remaining bone recently subjected to regenerative treatment has limited mechanical and vascular properties that imply a lesser osteointegration capacity in the first surgical step (6).

As to the regenerative technique used, the consulted literature indicates that the use of autologous bone is more predictable than the use of xenografts. However, it must be remembered that the use of auto-logous bone implies increased morbidity, since an additional surgical donor bed is needed $(7,8)$.

Modified ridge splitting technique for expansive dilatation of the vestibular and lingual crest walls affords a bone bed full of blood of marrow origin and great osteogenic potential that can facilitate the regenerative process without having to use autologous donor zones. Satisfactory results are thus ob-tained, with high predictability and low morbidity compared with those techniques that make use of autologous donor zones (9).

In addition, it must be remembered that regenerative techniques in general are inherently characterized by partial reabsorption of the initially created bone volume, as a result of bone remodeling and elastic-ity phenomena. In this sense, the present article describes the use of expansive corticotomy comple-mented with the application of new elements to prevent collapse of the expanded cortical bone (10).

Specifically, we use new conical threaded devices made of titanium and presenting a variable coronal diameter of 2, 3 and $4 \mathrm{~mm}$, referred to as space maintainers (Klockner $\left.{ }^{\circledR}\right)$. The latter are wedged be-tween the horizontally displaced crest walls, where they remain for the full period of horizontal ridge regeneration.

The clinical case presented in this study thus describes a new conservative surgical technique for the horizontal augmentation of highly atrophic alveolar ridges, without having to use autologous bone graft techniques that are characterized by more aggressive surgery and frequent secondary partial reab-sorption phenomena.

\section{Report of a Case}

A 56-year-old, non-smoking woman with no relevant history of disease presented with pain in the second quadrant, irradiating to the left eye and temple region, secondary to reactivation of chronic periapical infection in tooth 2.7 that had been endodontically treated $10-15$ years ago, and which in combination with tooth 2.3 acted as a post for a 5-tooth ceramometal bridge in that same quadrant (Fig. 1A).

The treatment plan involved the removal of tooth 2.7, the replacement of teeth 2.4 to 2.6 with im-plants, and the fitting of a new ceramometal crown on canine 2.3.

Due to important vertical and horizontal atrophy in the edentulous zone, the patient required prior sinus lift surgery and crestal widening with splitting technique and the placement of conical space maintainers (Klockner ${ }^{\circledR}$ ) to allow implant placement in a second surgical step with much more pre-dictable results.

Drug treatment fourteen days was administered. The pharmacological therapy was started four days before surgery, in the form of amoxicillin $1000 \mathrm{mg} / 8$ hours (the patient did not tolerate the combina-tion amoxici1 lin / clavulanate), ibuprofen $600 \mathrm{mg} / 8$ hours and $0.12 \%$ chlorhexidine rinse every 8 hours. Surgery was started, sectioning the ceramometal bridge distal to canine 2.3 (to temporarily pre-serve the crown). We then raised a full thickness flap to expose the edentulous ridge (measuring only $2.5 \mathrm{~mm}$ in thickness) and opened a window for access to the maxillary sinus using rotary instruments.

Before sinus filling with biomaterial, augmentation of the alveolar ridge adjacent to the sinus window was carried out, performing a corticotomy with rotary discs (Klockner ${ }^{\circledR}$ ) at 10,000-20,000 rpm and irrigating with abundant sterile solution (Fig. 1B).

The corticotomy consisted of deep sectioning at the center of the ridge (to the base of the sinus), with another vertical bone releasing section $1.5-2 \mathrm{~mm}$ in depth distal to tooth 2.3 .

These sections made it possible to partially luxate (green-stick fracture) the entire vestibular wall from the bone table, using threaded expanders and chisels to finally augment the alveolar ridge width to $6-7 \mathrm{~mm}$.

When starting bone expansion with this technique, preferably narrow chisels are recommended, which upon penetrating (by impacting) into the marrow space induce vestibular expansion of the oral cortical process. Depending on the extent of expansion, the number of implants to be placed, the bone density and the ridge profile (flat, convex or concave oral cortical layer), vertical releasing 


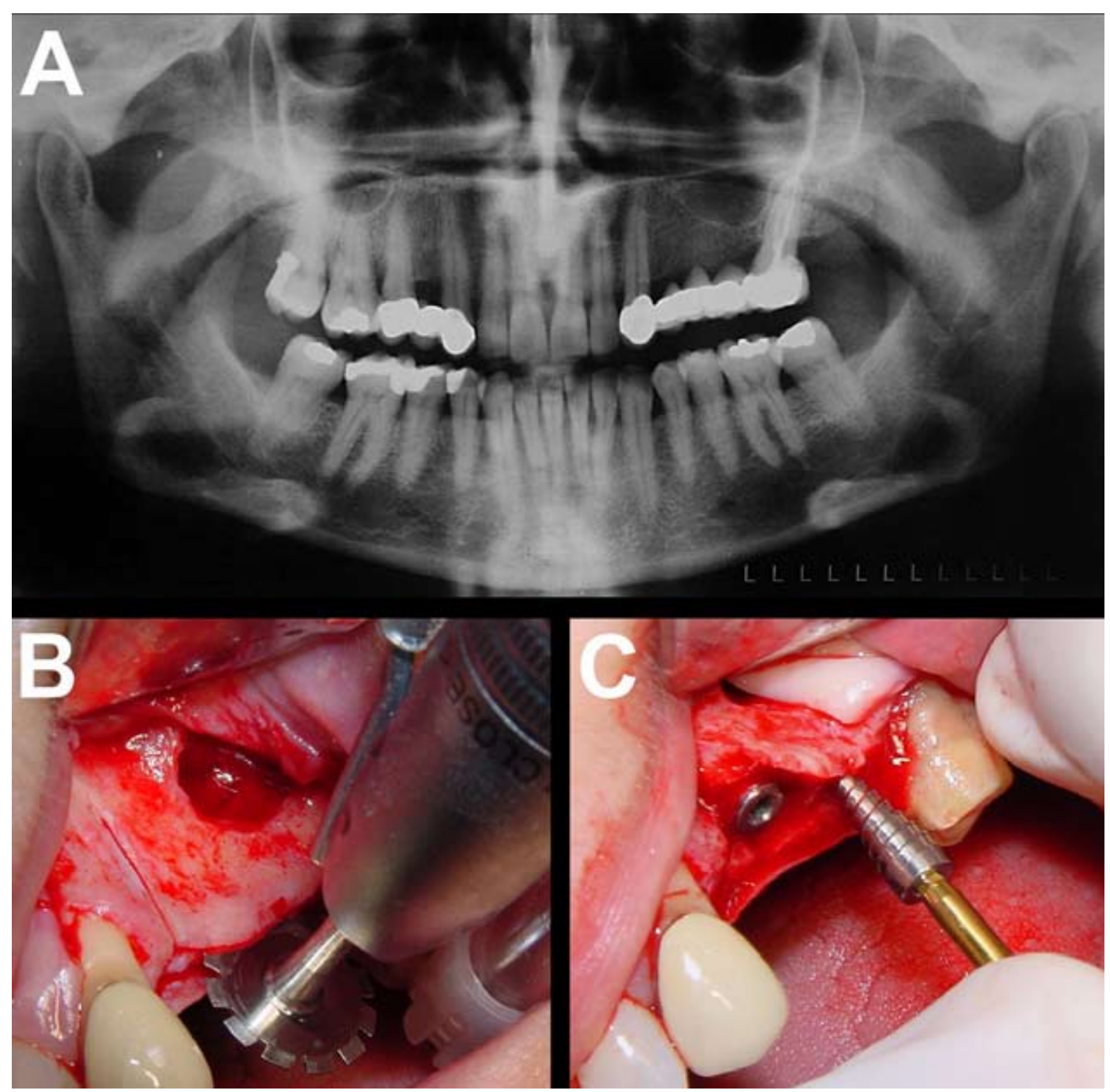

Fig. 1A. Preoperative panoramic X-ray view (chronic periapical infection of tooth 2.7).

Fig. 1B. Ostectomy for access to the maxillary sinus and crestal split using rotary discs.

Fig. 1C. Luxation (green-stick fracture) of the vestibular bone wall and placement of two threaded space maintainers osseous.

sections may prove necessary in the oral bone table at one or both extremes (mesial and distal) of the crestal corticotomy.

Once initial expansion was achieved with narrow chisels, threaded expanders of increasing diameter and conicity were inserted to increase the separation between the bone tables until the desired horizon-tal ridge dimension was obtained. In this patient the luxated cortical components were extremely thin and did not allow safe simultaneous placement of the implants. As a result, in the first surgical step we left inserted two conical threaded space maintainers osseous (Klockner ${ }^{\circledR}$ ) to preserve the ridge width achieved until the next surgical step (Fig. 1C).
These space maintainers are conical titanium screws that are highly polished in order to avoid osteoin-tegration, and are easily and fully inserted with the hexagonal driver. Their conical design allows easy placement and removal, together with maintenance of the cortical separation - preserving a maximum of free surrounding space at middle and apical level of the expanded ridge for filling with the blood clot that subsequently transforms into regenerated cancellous bone.

Thus, in our patient, after placing the two space maintainers, we filled the sinus cavity with reabsorb-able biomaterial. We then also added biomaterial to the surface of the space maintainers and displaced alveolar wall, with the purpose of homogenizing the bone contour of the 
operated zone. The biomate-rial used consisted of horse cancellous bone particles (BIO - GEN $\AA$, Bioteck $\left.{ }^{\circledR}\right)$ (particle size $0.5 \mathrm{~mm}$ and total reabsorption time 4-6 months) previously hydrated for 10 minutes with physiological saline to ensure easy transfer and adaptation to the surgical bed. Lastly, we placed a plasma rich in growth factors membrane over the graft and performed periosteal sections in the flap to increase its elasticity and thus secure primary closure of the surgical zone with tensionless sutures.

The procedure was completed with removal of tooth 2.7 together with careful curettage of its chronic periapical infection zone, and suturing of the socket. After surgery, the same drug treatment adminis-tered initially was maintained for a total of 10 additional days.

After a waiting period of 7 months to allow sinus and crest graft maturation (Fig. 2A), second step surgery was performed for placement of the implants. To this effect we again raised the full thickness flap, but in this case without extensive detachment apically, since only visualization of the space main-tainer heads was required (Fig. 2B). These heads were easily removed with the same hexagonal driver used to place them in the previous surgical step.

At this point neoformed bone of still limited density was observed, though presenting good vasculari-zation, with the two orifices created by the space maintainers. The implant sockets were prepared in these orifices using expanders and drills to secure in-depth penetration (Fig. 2C).

We positioned three implants measuring $3.8 \times 12 \mathrm{~mm}$ in size (Klockner ${ }^{\circledR}$ model SK-2), and finally added a

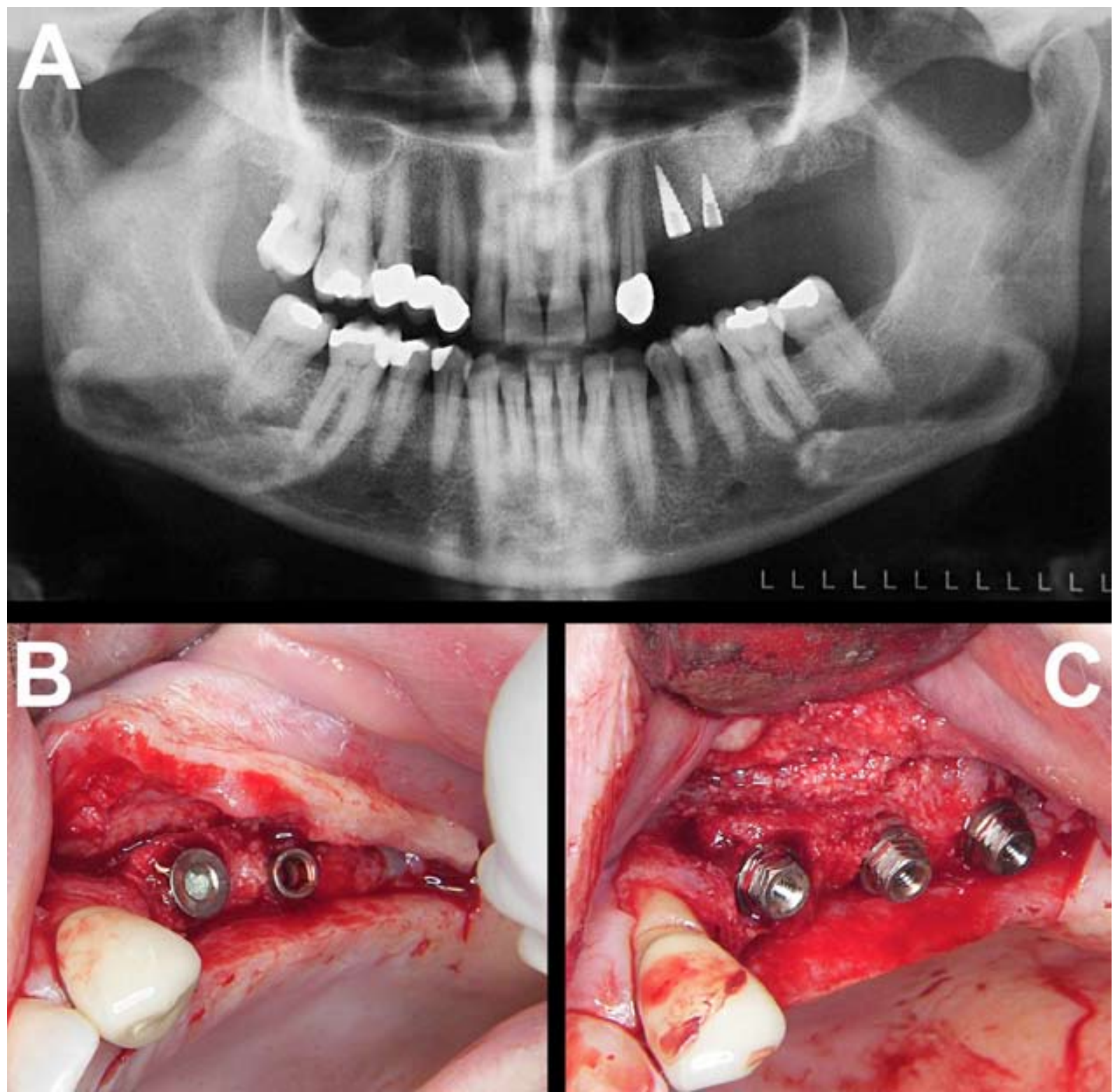

Fig. 2 A. Panoramic X-ray view showing the space maintainers 7 months after surgery.

Fig. 2 B-C. Second-step surgery for removal of the threaded maintainers and their replacement with three implants in the regenerated ridge. 
portion of biomaterial (BIO-GEN $\AA$, Bioteck $\AA$ ) over the small dehiscence in the more mesial implant (coinciding with the still immature vertical corticotomy zone), followed by wound suturing.

As has been commented above, due to the still quite immature neoformed bone observed at implant placement, we decided to allow a 6-month osteointegration period before carrying out the prosthodon-tic phase of treatment. After this period of time without complications, imprints were obtained simul-taneously from the implants and tooth 2.3 .

As can be seen in the final panoramic X-ray view (Fig. $3 \mathrm{~A}$ ), due to the close proximity of the apexes of canine 2.3 and implant 2.4, prior to definitive prosthesis placement we decided to perform preven-tive endodontic treatment of the canine, with a view to avoiding future retrograde bone alteration around the adjacent implant secondary to possible periapical infection of the canine. In this context, the canine had to be instrumented for the placement of a new ceramometal crown with the finishing line located $3 \mathrm{~mm}$ higher because of the antiesthetic gingival recession noted with the old bridge.

The corresponding ceramometal crowns were prepared in the laboratory: an independent crown with a ceramic shoulder for tooth 2.3, and three splinted crowns for the three implants. As implant abutments we used a machined titanium post for implant 2.4 and two manufactured composite posts for the other two implants (Fig. 3B). The posts were tightened in the mouth to a torque of $30 \mathrm{~N} \cdot \mathrm{cm}$, after, which the ceramometal crowns were cemented with composite cement (Panavia $\left.{ }^{\circledR}\right)$ (Fig. C).

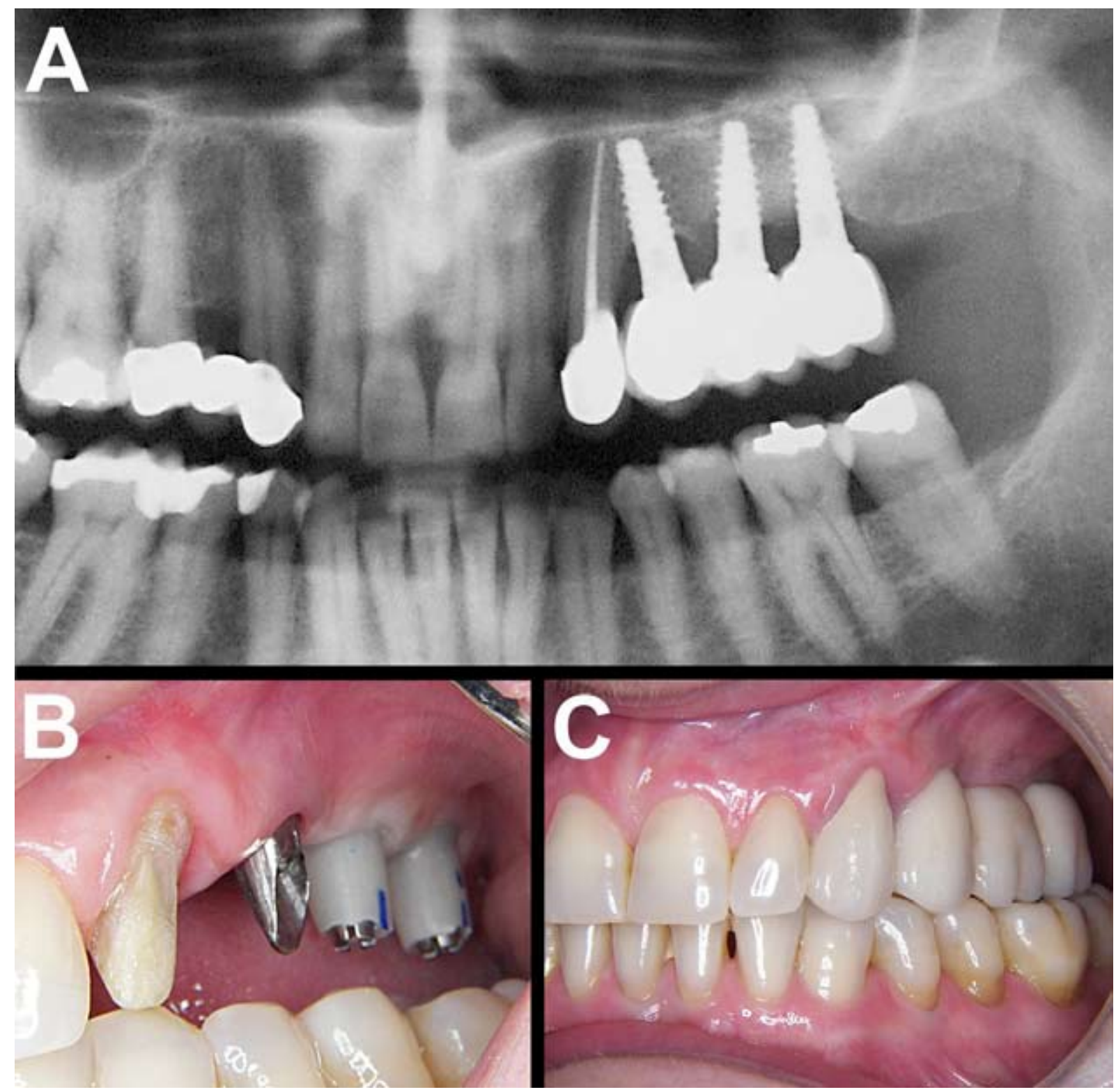

Fig. 3 A - C. Stable radiological and clinical condition 20 months after placing the definitive cemented ceramometal crowns.

Fig. 3 B. Detail of the posts used: a machined titanium post and two manufactured composite posts. 


\section{Discussion}

The use of bone threaded space maintainers osseous constitutes an interesting step in the evolution of the expansive corticotomy technique. In effect, they can improve the prognosis in cases of severe atro-phy where the ridge width is less than $3 \mathrm{~mm}$ and safe and predictable placement of a dental implant measuring 3.5-4 $\mathrm{mm}$ in diameter is complex and/or not feasible, fundamentally due to physiological reabsorption of the extremely thin remaining vestibular cortical component, which is further compro-mised by its limited vascularization (9). These circumstances make it necessary to delay implant placement following a first ridge augmentation step. Although this new technique is unable to shorten the implant placement waiting time (due to the inevi-table need for bone maturation), it avoids partial reabsorption of the neoformed bone during the men-tioned waiting period, and does not require the harvesting of autologous bone from other intra- or ex-traoral donor zones - thereby avoiding the increased morbidity associated with such procedures (10).

In addition, the present clinical case illustrates the importance of always placing the space thread maintainers considering the appropriate implant axis for the future prosthesis. If an adequate axis is not established, at the time of implant placement it probably will be very difficult or even impossible to correct the insertion axis initially established with a space maintainer positioned at an incorrect an-gle (11). This situation was seen in our case, since we were unable to correct the mesial inclination of the space maintainer in position 2.4 at the time of implant placement, due to the risk of fracturing the still immature bone through surgical maneuvering - particularly in the zone close to the vertical os-teotomy - and thus compromising the primary stability of the implant (Fig. 2).

While some authors (12) recommend placing the implants in neoformed bone between the space main-tainers instead of within the orifices created by them, the use of these orifices for implant placement simplifies the technique without negative consequences, since the surgical time is shortened and ma-nipulation of the still fragile neoformed bone is minimized.

As can be seen in the final panoramic X-ray view (Fig. $3 \mathrm{~A}$ ), due to the close proximity of the apexes of canine 2.3 and implant 2.4 , prior to definitive prosthesis placement we decided to perform preven-tive endodontic treatment of the canine, with a view to avoiding future retrograde bone alteration around the adjacent implant secondary to possible periapical infection of the canine. In this context, the canine had to be instrumented for the placement of a new ceramometal crown with the finishing line located $3 \mathrm{~mm}$ higher because of the antiesthetic gingival recession noted with the old bridge.

In this study we used two prosthetic composite abutments, which offer advantages and versatility with respect to the conventional prosthetic metal abutments (Fig. 3B). A long-term clinical study is being carried out with abutments of this type (13). Nevertheless, in the implant placed in position 2.4, a tita-nium post was used, which could offer thinner walls than a composite post, in order to compensate the lack of parallelism (over $20^{\circ}$ ) in the longitudinal axis of the implant (this being a limitation of compos-ite implants).

This modified technique made it possible for the threaded space maintainers osseous to avoid partial collapse of the initially obtained ridge widening, which tends to occur to one degree or other as a con-sequence of reabsorption during the physiological tissue repair process following ridge splitting tech-nique (9). In this way, at the time of second-step surgery for implant placement, the full ridge width obtained with the first-step expansive corticotomy was available.

\section{References}

1. Fiorellini JP, Nevins ML. Localizad ridge augmentation/preservation. A systematic review. Ann Periodontol. 2003; 8:321-7.

2. Aghaloo TL, Moy PK. Which hard tissue augmentation techniques are the most successful in furnishing bony support for implant placement? Int J Oral Maxillofac Implants. 2007; 22 Suppl:49-70.

3. Rocchietta I, Fontana F, Simion M. Clinical outcomes of vertical bone augmentation to enable dental implant placement: a systematic review. J Clin Periodontal. 2008; 35: 203-15.

4. Stevens B, Yang Y, Monadas A, Stucker B, Nguyen KT. A review of materials, fabrication methods, and strategies used to enhance bone regeneration in engineered bone tissues. J Biomed Mater Res B Appl Biomater. 2008; 85:573-82.

5. Kim SS, Kim BS. Comparison of osteogenic potential between apatite-coated poly(lactide-co-glycolide)/hydroxyapatite particles and Bio-Oss. Dent Mater J. 2008; 27:368-75.

6. Wallace SS, Froum SJ. Effect of maxillary sinus augmentation on the survival of endosseous dental implants. A systematic review. Ann Periodontol. 2003; 8: 328-43.

7. Cordaro L, Amadé DS, Cordaro M. Clinical results of alveolar ridge augmentation with mandibular block bone grafts in partially edentulous patients prior to implant placement. Clin Oral Implants Res. 2002; 13:103-11

8. Brugnami F, Caiazzo A, Leone C. Local intraoral autologous bone harvesting for dental implant treatment: alternative sources and criteria of choice. Keio J Med. 2009; 58:24-8.

9. Suh JJ, Shelemay A, Choi SH, Chai JK. Alveolar ridge splitting: a new microsaw technique. Int J Periodontics Restorative Dent. 2005; 25:165-71.

10. Chiapasco M, Ferrini F, Casentini P, Accardi S, Zaniboni M. Dental implants placed in expanded narrow edentulous ridges with the Extension Crest device. A 1-3 year multicenter follow-up study. Clin Oral Implants Res. 2006; 17: 265-72.

11. Lin CL, Wang JC, Ramp LC, Liu PR. Biomechanical response of implant system placed in the maxillary posterior region under various conditions of angulation, bone density, and loading. Int J Oral Maxillofac Implants. 2008; 23:57-64.

12. Funaki K, Takahashi T, Yamuchi K. Horizontal alveolar ridge augmentation using distraction osteogenesis: comparison with a bonesplitting method in a dog model. Oral Surg Oral Med Oral Pathol Oral Radiol Endod. 2009;107:350-8.

13. Andriani WJ, Suzuki M, Bonfante EA, Carvalho RM, Silva NR, Coelho PG. Mechanical testing of indirect composite materials directly applied on implant abutments. J Adhes Dent.2009 Nov 9: doi:10.3290/j.jad.a17710. 